1036 THE PROGNOSTIC ROLE OF BETA-CATENIN IN PATIENTS WITH ADVANCED STAGE SEROUS OVARIAN CANCER

II Aluloski", ${ }^{1} \mathrm{M}$ Tanturovski, ${ }^{2} \mathrm{~S}$ Kostadinova-Kunovska, ${ }^{2} \mathrm{R}$ Jovanovic, ${ }^{1} \mathrm{~V}$ Jovanovska, ${ }^{2} \mathrm{G}$ Petrushevska. ${ }^{1}$ University Clinic for Obstetrics and Gynecology-Skopje, Gynecologic Oncology, Skopje, Macedonia; ${ }^{2}$ Institute for Pathology. Medical Faculty-Skopje, Skopje, Macedonia

\subsection{6/ijgc-2021-ESG0.508}

Introduction/Background* Serous ovarian cancer is the most common sub-type of epithelial ovarian cancer and is the leading cause of cancer-related death among gynecologic cancer patients. Beta-catenin plays a vital role in the genesis of certain types of cancers. Its implications in the survival and prognosis of patients with serous ovarian cancer is not yet fully understood. The aim of the study was to analyze the association between beta-catenin expression, as well as certain other clinical and pathohistological characteristics of serous ovarian cancers, with the overall patient survival in advanced stage cases.

Methodology We conducted immunohistochemical analysis in tumor specimens from 40 patients to determine the expression of beta-catenin. We analyzed the relationship between beta-catenin expression and the FIGO disease stage and the tumor grade. We used Kaplan-Meier statistics to analyze the prognosis.

Result(s)* We detected increased expression of beta-catenin in patients with FIGO Stage III or IV $(p=0.0003)$. We did not detect a statistically significant association between beta-catenin expression and tumor grade $(p=0.817)$. The positive expression of beta-catenin was associated with shorter average survival $(p=0.034)$. There was no statistically significant relationship between beta-catenin expression and other pathohistological tumor features.

Conclusion* Beta-catenin expression is associated with poorer prognosis in patients with serous ovarian cancer.

\section{DIAGNOSTIC ACCURACY OF INTRAOPERATIVE FROZEN SECTION IN THE ASSESSMENT OF ADNEXAL TUMOURS}

${ }^{1} \mathrm{~A}$ Miguel, ${ }^{1} \mathrm{M}$ Plancha, ${ }^{1,2} \mathrm{D}$ Djokovic, ${ }^{1} \mathrm{PA}$ Duarte, ${ }^{1} \mathrm{C}$ Barros, ${ }^{1,3}{ }^{3}$ Pinto ${ }^{*}{ }^{1}$ Maternidade Dr. Alfredo da Costa - Centro Hospitalar Universitário de Lisboa Central, Lisboa, Portugal; ${ }^{2}$ Faculdade de Ciências Médicas, Nova Medical School, Lisboa, Portugal; ${ }^{3}$ First Faculty of Medicine Charles University, Prague, Czech Republic

\subsection{6/ijgc-2021-ESG0.509}

Introduction/Background* Intraoperative frozen section (IFS) is considered a relevant procedure to categorize adnexal lesions as benign, borderline or malignant. The aim of this study is to determine the accuracy of IFS performed in our tertiary referral centre by assessing its correlation with definitive histopathologic diagnosis.

Methodology This retrospective study included 89 patients with adnexal tumours, who consecutively underwent surgery and intraoperative histopathological examination in our Institution between 2017 and 2020. IFS was compared to definitive histopathological diagnosis and sensitivity, specificity, positive predictive value, negative predictive value and accuracy of IFS were determined according to malignancy status, with a $95 \%$ confidence interval (CI).

Result(s)* Mean patient age was 56.3 years (SD 14.3) and $53.9 \%(48 / 89)$ were post-menopausal. IFS indicated benign
Abstract 1042 Table 1 - IFS diagnostic value according to status of malignancy

\begin{tabular}{llll}
\hline Statistical value (\%) & $\begin{array}{l}\text { Benign } \\
(\mathrm{Cl} 95 \%)\end{array}$ & $\begin{array}{l}\text { Borderline } \\
(\mathrm{Cl} 95 \%)\end{array}$ & $\begin{array}{l}\text { Malignant } \\
(\mathrm{Cl} 95 \%)\end{array}$ \\
\hline Sensitivity & $94.8(85.6-98.8)$ & $75.0(45.8-94.5)$ & $76.47(50.1-93.2)$ \\
Specificity & $83.9(66.3-94.6)$ & $92.2(83.8-92.1)$ & $95.8(88.3-99.1)$ \\
Positive predictive value & $91.67(83.1-96.1)$ & $60.0(39.4-77.6)$ & $81.3(58.1-93.1)$ \\
Negative predictive value & $89.7(74.0-96.4)$ & $96.0(89.9-98.4)$ & $94.5(88.0-97.6)$ \\
Accuracy & $91.0(83.1-96.0)$ & $89.9(81.7-95.3)$ & $92.1(84.5-96.8)$ \\
\hline
\end{tabular}

tumours in 60 cases (67.4\%), borderline in $12(13.5 \%)$ and malignant in 17 (19.1\%). Definitive histopathologic assessment diagnosed benign tumours in 58 cases $(65.2 \%)$, borderline in $15(16.9 \%)$ and malignant in 16 cases (18\%). Concordance between IFS and definitive histopathologic diagnosis was found in 77 cases (86.5\%). Overall accuracy of IFS was $92.1 \%$ for malignant tumours, $91.0 \%$ for benign and $89.9 \%$ for borderline.

Conclusion* In our series of patients, in agreement with previously published data, IFS was an important tool in the assessment of adnexal masses, allowing an adequate surgical staging of invasive malignancies. With low positive predictive value, IFS presented limitations in the diagnosis of borderline tumours.

\section{THE DEVELOPMENT OF AN INTERNATIONAL REGISTRY WITH IMPLEMENTATION OF A DELPHI PROCESS AROUND THE ROLE OF HIPEC IN OVARIAN CANCER}

${ }^{1} \mathrm{C}$ Thompson*, ${ }^{2} \mathrm{~W}$ Van Driel, ${ }^{3} \mathrm{~L}$ Perrin, ${ }^{3} \mathrm{~S}$ Barry, ${ }^{4} \mathrm{O}$ Zivanovic, ${ }^{5} \mathrm{~B}$ Moran, ${ }^{1} \mathrm{D}$ Brennan. ${ }^{1}$ Mater Misericordiae University Hospital, Gynaecological Oncology, Dublin, Ireland; ${ }^{2}$ The Netherlands Cancer Institute , Amsterdam; ${ }^{3}$ Mater Hospital Brisbane, Brisbane, Australia; ${ }^{4}$ Memorial Sloan Kettering, New York, USA; ${ }^{5}$ Basingstoke Peritoneal Malignancy Institute, Basingstoke, UK

\subsection{6/ijgc-2021-ESG0.510}

Introduction/Background* The role of HIPEC in ovarian cancer has been the subject of significant debate. The publication of the OVIHIPEC trial has provided evidence to support the use of HIPEC in the interval cytoreduction setting with a complete or near partial macroscopic resection ${ }^{1}$.

It is clear from many of the recent surgical trials in advanced and recurrent ovarian cancer that the international gynaecological oncology community has not been successful in implementing standardised surgical approaches to advanced ovarian cancer cytoreductive surgery (CRS). This includes the role, accreditation and operational protocols for HIPEC.

Methodology Our aim was to ascertain the global interest of centres practising CRS for ovarian cancer and HIPEC of contributing to an International Data Registry with a view to implement a Delphi process to address these vital issues in ovarian cancer surgery. A SurveyMonkey invitation was distributed to centres already involved within the Peritoneal Surface Oncology Group International (PSOGI) database. Invitation was voluntary and consent obtained to become involved with the registry. A short 5 question survey was initially issued to assess referral levels and accreditation requirements. 
Result(s)* 40 of 98 centres replied to the survey. $78 \%$ consented to become involved in an international registry. $63 \%$ of centres who replied receive between $50->100$ referrals of ovarian cancer per year and $90 \%$ perform HIPEC in ovarian cancer. $79 \%$ (31/40) stated they had been practising CRS \& HIPEC for $>10$ years. The number of CRS with HIPEC per year performed was $<20$ cases in $56 \%$ of respondents and $>50$ in $4 \%$.

$21 \%$ of centres held international accreditation for ovarian cancer CRS and 51\% held national accreditation. Of interest was that $82 \%$ reported that no certification was required for the administration of HIPEC in their country.

Conclusion* Given the multiple reports demonstrating variations in practice across the globe we believe this could be a very important opportunity for implementing change for women with ovarian cancer. For centres that consented to participation a further survey will be issued focusing on operating standards for CRS in ovarian cancer and protocols for HIPEC administration.

\section{BLOOD-BASED DETECTION OF CIRCULATING DICKKOPF- 1 AS A PROGNOSTIC BIOMARKER IN OVARIAN CANCER PATIENTS}

1,2 $\mathrm{P}$ Wimberger*, ${ }^{1,2} \mathrm{DM}$ Klotz, ${ }^{1,2} \mathrm{~T}$ Link, ${ }^{1,2} \mathrm{M}$ Goeckenjan, ${ }^{3} \mathrm{~N}$ Jaschke, ${ }^{3} \mathrm{LC}$ Hofbauer, ${ }^{3}$ A Göbel, ${ }^{3}$ TD Rachner, ${ }^{1,2} \mathrm{JD}$ Kuhlmann. ${ }^{1}$ Technische Universität Dresden, Department of Gynecology and Obstetrics, Dresden, Germany; ${ }^{2}$ National Center for Tumor Diseases, Partner site Dresden (NCT), Dresden, Germany; ${ }^{3}$ Technische Universität Dresden, Division of Endocrinology, Diabetes, and Bone Diseases, Department of Medicine III

\subsection{6/ijgc-2021-ESG0.511}

Introduction/Background* Dickkopf-1 (DKK-1) is a secreted protein, known for suppressing the differentiation and activity of bone-building osteoblasts by acting as an inhibitor of Wntsignalling. Soluble DKK-1 (sDKK-1) has been proposed as prognostic biomarker for a wide range of malignancies, however, clinical relevance of sDKK-1 as potential blood-based marker for ovarian cancer is unknown.

Methodology sDKK-1 levels were quantified in a cohort of 150 clinically documented ovarian cancer patients by a commercially available DKK-1 ELISA (Biomedica, Vienna, Austria). Result(s)* Median sDKK-1 level was significantly elevated at primary diagnosis of ovarian cancer compared to healthy controls (estimated difference (ED) of $7.75 \mathrm{ng} / \mathrm{mL}$ (95\%CI: 3.01 - $12.30 \mathrm{ng} / \mathrm{mL}, \mathrm{p}=0.001)$ ). Higher levels of sDKK-1 at diagnosis indicated an increased volume of intraoperative malignant ascites (ED $7.08 \mathrm{pmol} / \mathrm{L}, 95 \% \mathrm{CI}$ : 1.46 - 13.05, p $=0.02$ ) and predicted suboptimal debulking surgery (ED 6.88 pmol/L, 95\%CI: 1.73 - 11.87, p = 0.01). sDKK-1 did not correlate with CA125, and higher sDKK-1 levels predicted a higher risk of recurrence and poor survival (PFS: HR = $0.507,95 \% \mathrm{CI}: 0.317-0.809 ; \mathrm{p}=0.004$; OS: $\mathrm{HR}=0.561$, 95\%CI: $0.320-0.986 ; p=0.044)$. Prognostic relevance of sDKK-1 was partly sustained in wtBRCA patients (PFS: HR = $0.507,95 \%$ CI: $0.317-0.809$; $\mathrm{p}=0.004)$.

Conclusion* This is the first study demonstrating the prognostic relevance of sDKK-1 in ovarian cancer patients, including those with wtBRCA1/2 status. Our data encourage further evaluation of sDKK-1 in ovarian cancer patients, possibly in terms of a therapy monitoring marker or a response predictor for sDKK-1-directed targeted therapies.

\section{ROLE OF CA 125, HE4 AND IOTA SIMPLE RULES IN DIFFERENTIATING BENIGN AND MALIGNANT OVARIAN TUMOUR}

A Bahadur, R Mundhra*, N Bhattacharya. aiims rishikesh, obs gynae, rishikesh, India

\subsection{6/ijgc-2021-ESG0.512}

Introduction/Background* This study aimed to evaluate the diagnostic added-value of serum CA-125 \& HE4 to the International Ovarian Tumor Analysis (IOTA) Simple Rules for differentiating malignant and benign ovarian tumors preoperatively.

Methodology A Cross sectional study was conducted in the Department of Obstetrics and Gynecology on 100 patients with ovarian masses. Demographic and clinical data were prospectively collected. Histopathologic diagnosis was used as the gold standard test. Evaluation of the utility of HE4, CA125 and IOTs simple rules for preoperative identification of malignacy was based on the increment of the area under the receiver operating characteristic curve (AuROC).

Result(s)* Out of the 50 suspected benign masses as per IOTA simple rules, post surgery (considering histopathology as gold standard) $32(64 \%)$ were diagnosed as benign ovarian masses, $4(8 \%)$ borderline and $14(28 \%)$ other malignant ovarian tumors on final histopathology. Out of 50 suspected malignant masses as per IOTA simple rules, 26 (52\%) were finally diagnosed as benign, 4(8\%) borderline and $20(40 \%)$ were malignant. Sensitivity of combined testing of both tumour markers (HE4 and Ca-125) and IOTA together was calculated to be a $85.3 \%(69-95)$ whereas its specificity increased to be $94.8 \%$ (86-99). Positive predictive value was determined to $90.6 \%$ (75-98) and negative predictive value was calculated $91.7 \%$ (82-97) making its diagnostic accuracy as 91.3\% (84-96).

Conclusion* Combining IOTA Simple rules with knpown tumour markers (CA125 and HE4) increases diagnostic accuracy in predicting malignat ovarian masses preoperatively

\section{BRCA GERMLINE MUTATION FREQUENCY AND THE EFFECTS ON ONCOLOGIC OUTCOMES AMONG NORWEGIAN OVARIAN CANCER PATIENTS}

\begin{abstract}
${ }^{1}$ A Birgisdottir*, ${ }^{2} \mathrm{M}$ Bjørnslett, ${ }^{3} \mathrm{~S}$ Aaserud, ${ }^{1} \mathrm{~T}$ Paulsen, ${ }^{1} \mathrm{~A}$ Dørum. ${ }^{1}$ The Norwegian Radium Hospital, Department of Gynecological Oncology, Oslo, Norway; ${ }^{2}$ Oslo University Hospital, Department of Molecular Oncology, Oslo, Norway; ${ }^{3}$ The Norwegian Cancer Registry, Oslo
\end{abstract}

\subsection{6/ijgc-2021-ESG0.513}

Introduction/Background* Germline mutations in the BRCA1 and $B R C A 2$ genes (BRCAg) are a known risk factor for development of breast and epithelial ovarian cancer (OC). We describe the frequency of $B R C A g$ carriers, the clinical features and survival in Norwegian OC patients.

Methodology This is a prospective cohort study including OC patients at the Norwegian Radium Hospital (covering 50\% of the Norwegian population) from Jan 1st 2014 to Dec 31st 2019. Included are the patients which accepted BRCAg test after giving informed consent. DNA was isolated from peripheral blood, and mutation analyses performed with Sanger sequencing and multiplex ligation probe amplification. Data on family history, BRCAg test results was registered and data on clinical features and histopathology was collected from the department's quality database. All statistical analyses were performed using the Stata (Stata/MP 17.1) program, Chi-square 